\title{
Bitte um Kandidatenvorschläge für den Vorstand 2013-2015
}

Liebe Mitglieder der Deutschen Röntgengesellschaft,

ich darf Sie hiermit aufrufen, Kandidatenvorschläge für den am 30. Mai 2013 zu wählenden Vorstand bis zum 15. April 2013 bei der Geschäftsstelle der DRG (Ernst-Reuter-Platz 10, 10587 Berlin) schriftlich einzureichen. Die Liste aller bis zu diesem Termin vorgeschlagenen Kandidaten wird zu Beginn des Röntgenkongresses veröffentlicht, sodass für die Mitglieder genügend Zeit besteht, sich mit den Wahlvorschlägen vertraut $\mathrm{zu}$ machen. Hiervon bleibt das Recht, auf der Mitgliederversammlung bis zum Beginn der Wahl weitere Kandidaten vorzuschlagen, unberührt.
Auf der Mitgliederversammlung am 30. Mai 2013 sind nach §8 (1) der Satzung der Deutschen Röntgengesellschaft zu wählen:

- Präsident der DRG für die Amtszeit 2015-2017

für die Amtszeit 2013-2015:

- Schatzmeister

- Schriftführer

- Vorstandsmitglied (Niedergelassene Radiologen)

- Vorstandsmitglied (Neuroradiologen)

- Vorstandsmitglied (Krankenhausradiologen) 
- Vorstandsmitglied (Pädiatrischer Radiologen)

- Vorstandsmitglied (Naturwissenschaftler)

Bereits in den Vorstand gewählt sind der ausscheidende Präsident (Prof. Forsting) und der für die Amtszeit 2013-2015 gewählte Präsident (Prof. Hosten). Jedes Mitglied der DRG ist berechtigt, Kandidaten zu benennen, wie auch jedes Mitglied der DRG berechtigt ist, für ein Vorstandsamt zu kandidieren.

Bitte fügen Sie Ihrem Vorschlag die Erklärung bei, dass die von Ihnen vorgeschlagene(n) Person(en) zugestimmt hat (haben), bei der Wahl für das Vorstandsamt zu kandidieren. Wahlvorschläge, bei denen diese schriftliche Erklärung fehlt, können nicht berücksichtigt werden.

Prof. Dr. Michael Forsting, Präsident 